\title{
An evaluation of differential susceptibility of barley varieties to lodging under varying environmental conditions
}

\author{
T. HURME*, J. ÖFVERSTEN AND L. JAUHIAINEN \\ Research Services, MTT Agrifood Research Finland, FIN-31600 Jokioinen, Finland \\ (Revised MS received 21 June 2006; First published online 16 August 2006)
}

\begin{abstract}
SUMMARY
Information on the variation in susceptibility to lodging between barley varieties under different environments can be used for local prediction. It can also be used to identify varieties that are robust to environmental variation. Efficient methods to obtain analogous information on the local yielding capacity of crop varieties have recently been established. The present paper extends the use of these methods for the analysis of dichotomously measured performance characteristics, the susceptibility to lodging, in particular. The procedures examined were based on generalized linear models in which the expected conditional mean of the susceptibility to lodging was used as an explanatory variable to express the environmental effects of a given environment. Through the use of logistic transformation the approach extends previous methods developed for continuous response variables to binary response variables. Models were subsequently used to obtain measures of susceptibility to lodging of each barley variety in terms of environmental variation. While the emphasis is restricted to lodging, similar methods can also be applied to other performance characteristics.
\end{abstract}

\section{INTRODUCTION}

The susceptibility of barley to lodging changes as a function of environmental conditions. In the current study, the susceptibility among varieties under different environments was examined, in order to establish whether individual barley varieties respond differently to environmental changes. Previous reports have referred to the variable susceptibility to lodging as a sensitivity that changes in response to differing environmental conditions (e.g. Finlay \& Wilkinson 1963).

Differences in lodging between barley varieties are relevant for two main reasons. Firstly, when environmental conditions are predictable, it is possible to select varieties that perform well in those particular conditions. Secondly, if environmental conditions are not predictable, it is possible to minimize risks by choosing varieties that are robust to environmental variation.

When varieties are selected for the Finnish national list of recommended varieties three criteria are

* To whom all correspondence should be addressed. Email: timo.hurme@mtt.fi used: crop yield, disease resistance and susceptibility to lodging, with yield potential being the most important. Consequently, the yield potential of barley varieties has been widely researched and the methods used are well defined. Yield is measured as a continuous variable. Such measurements can usually be assumed to be normally distributed and therefore a number of well-established methods can be used to analyse crop yield data. The two most widely used methods are linear regression and linear mixed models. These methods have also been extended to evaluate the variation in sensitivity to environmental conditions amongst barley varieties. Early approaches were based on linear regression (Yates \& Cochran 1938; Finlay \& Wilkinson 1963; Eberhart \& Russell 1966). Oman (1991) and Gogel et al. (1995) were the first to apply linear mixed models to evaluate genotype $\times$ environment interactions. Piepho (1997, 1998, 1999) has clarified and extended the methods and presented well-established approaches to estimate the sensitivity parameters required. Öfversten (1998) and Öfversten et al. (2002) have used multiplicative linear mixed models to assess and predict the local performance of crop varieties, using a conditional mean of crop yield given the environment 
as a measure of the natural environment. The conditional mean combines all environmental effects into a single environmental indicator. This is useful, since characterization of environmental conditions using well-defined variables is difficult.

To study the variation in susceptibilities to lodging among barley varieties, data originating from Finnish statutory variety trials were used. In addition to yield measurements, these data include measurements of lodging and disease resistance. Lodging is visually assessed by estimating the proportion of lodging in the field. Such measurements cannot be assumed to be normally distributed and therefore a different analysis has to be applied from that used for yield data, which does follow a normal distribution. Fortunately, recent statistical theory and software has been sufficiently developed to allow efficient analysis of non-normal data. This permitted a more thorough analysis of existing data of lodging of barley varieties using a logistic model otherwise analogous to that used by Piepho (1997) and Öfversten et al. (2002).

The objective of the present study was to investigate the susceptibility of barley varieties to lodging under variable environmental conditions. The results are intended to be useful for producers in the selection of barley varieties to be grown under local conditions. In such situations, producers typically have some indication of the risks of lodging in their fields, based on earlier experiences. The current results assist producers in making the best use of existing knowledge.

After determining the parameters describing the susceptibility to lodging, their correlation with the physical properties of varieties was straightforward to calculate. Determining such estimates was not necessary for the main objectives of the study but was conducted to complement the general knowledge on the subject and verify our findings with previous data reported in the literature.

\section{MATERIALS AND METHODS}

The data used originate from statutory variety trials conducted in Finland by MTT Agrifood Research Finland. Such data have been collected over the past 30 years. Currently, the trials are carried out at some 20 experimental sites and the annual number of single trials exceeds 100 . The experimental plots used in trials are usually arranged according to an incomplete block design with three or four replicates. As response variables, estimated trial wise means were used to characterize the performance of individual barley varieties.

The original data included more than 300 barley varieties, most of which were not cultivated in recent years. Also, some new varieties did not have enough observations to be included in the statistical computations; therefore, only varieties assessed in more than
35 trials were selected. However, a few exceptions were made. Varieties Edel, Botnia and Erkki were included in the analysis even though they had fewer than 35 observations (32, 26 and 29, respectively), since earlier studies (Kangas et al. 2005) indicated that these had interesting lodging properties. The varieties examined are all commonly cultivated in Finland and therefore formed a representative sample of Finnish barley varieties. Furthermore, varieties Kunnari and Scarlett have been commonly used as reference varieties in variety testing and therefore form convenient benchmarks for producers when examining the results of the present study. In compliance with the common analysis of variety trial data (Kangas et al. 2005), only data from the last 8 years (1997-2004) were evaluated. Consequently, analysis was performed on data from 14 experimental sites, 8 years and the 14 most commonly grown barley varieties (Table 1).

In the data used, lodging was recorded by visual assessment of the proportion of lodging in the field. Preliminary investigation showed that these measurements were inaccurate and rather subjective. Furthermore, the distribution of lodging appeared to be highly dependent on the observer. To retain most of the information, while removing subjective elements, lodging recordings were transformed into binary scale. In most cases, an observed score above $0 \cdot 10$ was consistent with a commercially significant amount of lodging, whereas lodging measures below $0 \cdot 10$ appeared to be irrelevant. Therefore, a limit of $0 \cdot 10$ was used for assigning lodging into a binary variable.

Logistic regression is the standard approach used to analyse binary probabilities (McCullagh \& Nelder 1989; Collett 2003; Agresti 2002; Cox \& Snell 1989; McCulloch \& Searle 2001). In logistic regression, the probability scale is first transformed from the range $(0,1)$ to $(-\infty, \infty)$ using a logistic transformation (logit), where $\operatorname{logit}(p)=\log \{p /(1-p)\}$. A linear model is then adopted for the transformed value of the success probability. The procedure ensures that the fitted probabilities lie between zero and unity (Collett 2003).

As a preliminary investigation of the prevalence of variety $\times$ environment interaction, a logistic analogy of the technique first used by Yates \& Cochran (1938) was applied. The analysis confirmed the occurrence of variety $\times$ environment interaction in the current data. This finding justified further exploration and evaluation of experimental data with more refined multiplicative models.

Öfversten et al. (2002) have shown that multiplicative mixed linear models serve as efficient tools in assessing and predicting the local yielding performance of wheat varieties. Such models are statistically sound and usually fit the data well. The models include environmental scores as explanatory 
Table 1. Summary of experimental data*

\begin{tabular}{lclccc}
\hline \hline $\begin{array}{l}\text { Barley } \\
\text { variety }\end{array}$ & $\begin{array}{c}\text { Number of } \\
\text { observations }\end{array}$ & \multicolumn{1}{c}{ Years } & $\begin{array}{c}\text { Growing } \\
\text { Cycle (days) }\end{array}$ & $\begin{array}{c}\text { Height } \\
(\mathrm{cm})\end{array}$ & $\begin{array}{c}\text { Lodging } \\
\text { (proportion } \\
\text { of plot area) }\end{array}$ \\
\hline Edel & 32 & $2001,2003-04$ & $90 \cdot 8$ & $78 \cdot 8$ & $0 \cdot 11$ \\
Optima & 46 & $1997-2004$ & $95 \cdot 2$ & $61 \cdot 8$ & $0 \cdot 12$ \\
Saana & 90 & $1997-2004$ & $89 \cdot 8$ & $66 \cdot 7$ & $0 \cdot 15$ \\
Tofta & 48 & $1997-2002$ & $93 \cdot 4$ & $65 \cdot 8$ & $0 \cdot 17$ \\
Kunnari & 89 & $1997-99,2001-04$ & $89 \cdot 0$ & $77 \cdot 0$ & $0 \cdot 22$ \\
Scarlett & 71 & $1997-2004$ & $91 \cdot 8$ & $64 \cdot 8$ & $0 \cdot 23$ \\
Kustaa & 65 & $1997-2001$ & $91 \cdot 5$ & $70 \cdot 8$ & $0 \cdot 26$ \\
Gaute & 40 & $2001-04$ & $86 \cdot 2$ & $79 \cdot 3$ & $0 \cdot 26$ \\
Arve & 100 & $1997-2003$ & $83 \cdot 5$ & $77 \cdot 8$ & $0 \cdot 28$ \\
Jyvä & 60 & $1997-2004$ & $86 \cdot 9$ & $73 \cdot 8$ & $0 \cdot 28$ \\
Rolfi & 38 & $1998-2000,2004$ & $83 \cdot 0$ & $70 \cdot 9$ & $0 \cdot 32$ \\
Erkki & 29 & $1997-2000,2002-03$ & $86 \cdot 8$ & $75 \cdot 7$ & $0 \cdot 37$ \\
Pohto & 72 & $1997-2001$ & $87 \cdot 5$ & $69 \cdot 9$ & $0 \cdot 39$ \\
Botnia & 26 & $1997-99,2003$ & $88 \cdot 5$ & $75 \cdot 6$ & $0 \cdot 48$ \\
\hline \hline
\end{tabular}

* Derived from Kangas et al. (2005).

variables. Environmental scores are latent variables that combine the effects of all the environmental conditions associated with a given experiment.

In the current study, the multiplicative mixed linear model approach of Piepho (1997) and Öfversten et al. (2002) was combined with the traditional logistic regression approach, i.e. a logistic transformation was incorporated into the multiplicative mixed linear model. This model combines the good local prediction properties of the multiplicative mixed linear model with the ability of the logistic model to handle binary data.

The following logistic multiplicative mixed linear model was used to analyse the susceptibility to lodging:

$$
\operatorname{logit}\left(p_{i j}\right)=g_{i}+b_{i} w_{j}+d_{i j}
$$

where $p_{i j}$ is the probability of lodging of variety $i$ in environment $j, g_{i}$ and $b_{i}$ are fixed parameters related to variety $i, w_{j}$ is normally distributed latent variable related to environment $j$ and $d_{i j}$ is a random deviation (unexplained interaction). The $w_{j}$ and $d_{i j}$ are assumed to be mutually independent and variances of $w_{j}$ and $d_{i j}$ are denoted by $\sigma_{w}^{2}$ and $\sigma_{d}^{2}$, respectively. The model in Eqn (1) is overparameterized which complicates the estimation of model parameters $g_{i}$ and $b_{i}$. Using the estimation procedure demonstrated by Piepho (1997) for an analogous linear mixed model, however, the GLIMMIX procedure of SAS 9 can be used (SAS Institute, Cary, NC) to obtain estimates $\hat{g}_{i}$ and $\lambda_{i}$ for the general mean parameter $g_{i}$ and the composite multiplicative parameter $\lambda_{i}=b_{i} \sigma_{w}$ and, furthermore, imposing a natural restriction $\bar{b} .=1$, obtain estimates $\hat{b}_{i}$ for the parameters $b_{i}$ as $\hat{b}_{i}=\hat{\lambda}_{i} / \bar{\lambda}$. The approximated standard errors for $\hat{b}_{i}$ were obtained from the inverse of the Fisher information matrix (Piepho 1997).

The model in Eqn (1) expresses the probability of lodging of variety $i$ in terms of an environmental latent variable $w_{j}$. Since $w_{j}$ is unobservable, this model cannot be used for predictive purposes. However, it is possible to modify Eqn (1) so that $w_{j}$ will be replaced by the respective mean environmental probability to lodging $p_{j}$. The resulting model can be used for prediction since $p_{j}$ is observable and its value at any environment of interest can be assessed from the previous cultivation history of the given environment.

It therefore follows that Eqn (1) can be rewritten as:

$$
\hat{p}_{i j}=\hat{p}_{i}\left(w_{j}\right)=\frac{e^{\hat{g}_{i}+\hat{b}_{i}\left(w_{j}\right)}}{1+e^{\hat{g}_{i}+\hat{b}_{i}\left(w_{j}\right)}}
$$

where $\hat{p}_{i j}$ is the estimated probability of variety $i$ to become lodged in environment $j$. Replacing the parameters $\hat{g}_{i}$ and $\hat{b}_{i}$ by their mean values yields:

$$
\hat{p}_{j}=\hat{p}\left(w_{j}\right)=\frac{e^{\bar{g} \cdot+w_{j}}}{1+e^{\bar{g} \cdot+w_{j}}}
$$

where $\hat{p}_{j}$ can be interpreted as the predicted mean probability of lodging at environment $j$ averaged over all the varieties tested in the study. Combining this with the fact that the trial data used is a representative sample of all the varieties studied and the environments of interest imply that $\hat{p}_{j}$ is a consistent estimate of the respective theoretical expected value. From this the following expression is given for the latent environmental variable $w_{j}$ :

$$
w_{j}=\log \left(\frac{\hat{p}_{j}}{1-\hat{p}_{j}}\right)-\bar{g} .
$$


Table 2. Estimates of $\hat{g}_{i}$ and $\hat{b}_{i}$ values for individual barley varieties

\begin{tabular}{|c|c|c|c|c|c|c|}
\hline $\begin{array}{l}\text { Barley } \\
\text { variety }\end{array}$ & $\hat{g}_{i}$ & S.E. $\left(\hat{g}_{i}\right)$ & $\begin{array}{c}P \text {-value* } \\
\left(H_{0}: g_{i}=\bar{g} .\right)\end{array}$ & $\hat{b_{i}}$ & S.E. $\left(\hat{b_{i}}\right)$ & $\begin{array}{c}P \text {-value* } \\
\left(H_{0}: b_{i}=\bar{b} .=1\right)\end{array}$ \\
\hline Arve & $0 \cdot 052$ & $0 \cdot 1937$ & 0.002 & $1 \cdot 001$ & 0.0931 & 0.496 \\
\hline Botnia & $0 \cdot 387$ & $0 \cdot 3245$ & 0.003 & $1 \cdot 149$ & $0 \cdot 1733$ & $0 \cdot 195$ \\
\hline Edel & -1.745 & $0 \cdot 4250$ & 0.002 & $1 \cdot 192$ & $0 \cdot 1683$ & $0 \cdot 127$ \\
\hline Erkki & -0.013 & $0 \cdot 2945$ & 0.047 & $1 \cdot 039$ & $0 \cdot 1539$ & 0.400 \\
\hline Gaute & $-0 \cdot 325$ & $0 \cdot 2690$ & $0 \cdot 251$ & $1 \cdot 056$ & $0 \cdot 1451$ & $0 \cdot 349$ \\
\hline Jyvä & -0.419 & $0 \cdot 2146$ & $0 \cdot 344$ & $0 \cdot 840$ & $0 \cdot 1134$ & 0.079 \\
\hline Kunnari & -0.595 & $0 \cdot 2060$ & $0 \cdot 332$ & 0.976 & 0.0925 & $0 \cdot 398$ \\
\hline Kustaa & -0.567 & $0 \cdot 2252$ & $0 \cdot 393$ & 0.969 & $0 \cdot 1093$ & $0 \cdot 389$ \\
\hline Optima & -1.686 & $0 \cdot 3276$ & $<0.001$ & 0.923 & $0 \cdot 1317$ & $0 \cdot 280$ \\
\hline Pohto & 0.053 & $0 \cdot 2136$ & 0.004 & $1 \cdot 006$ & $0 \cdot 1068$ & 0.479 \\
\hline Rolfi & $0 \cdot 040$ & $0 \cdot 2597$ & 0.018 & 0.960 & $0 \cdot 1305$ & $0 \cdot 381$ \\
\hline Saana & $-1 \cdot 100$ & $0 \cdot 2220$ & 0.004 & 0.933 & 0.0927 & $0 \cdot 236$ \\
\hline Scarlett & -0.662 & $0 \cdot 2255$ & $0 \cdot 244$ & $1 \cdot 003$ & $0 \cdot 1014$ & 0.487 \\
\hline Tofta & -0.497 & $0 \cdot 2450$ & $0 \cdot 487$ & 0.952 & $0 \cdot 1279$ & $0 \cdot 354$ \\
\hline
\end{tabular}

* $P$ values are obtained using approximate Wald tests. For each $\hat{g}_{i}$ the test shows the probability that the true value of the respective parameter equals the mean of all the $g_{i}$ parameters. For each $\hat{b}_{i}$ the test shows the probability that the true value of the respective parameter equals the mean of all the $b_{i}$ parameters.

Inserting Eqn (4) into Eqn (2) allows the probability of variety $i$ to become lodged in environment $j$ to be expressed as:

$$
\hat{p}_{i j}=\frac{e^{\hat{g}_{i}+\hat{b}_{i}\left[\log \left(\frac{\hat{p}_{j}}{1-\hat{p}_{j}}\right)-\bar{g} .\right]}}{1+e^{\hat{g}_{i}+\hat{b}_{i}\left[\log \left(\frac{\hat{p}_{j}}{1-\hat{p}_{j}}\right)-\bar{g} .\right]}} .
$$

The above formula can be used to generate graphs in which the susceptibility to lodging of variety $i$ is presented as a function of the mean probability of lodging in environment $j$. Such graphs can be used yet further to predict the susceptibility to lodging of a given variety with respect to environmental conditions.

\section{RESULTS}

The estimated parameters $\hat{g}_{i}$ and $\hat{b}_{i}$, their respective standard errors and test statistics comparing individual parameters to their respective means are presented in Table 2. Equation (5) and the estimated parameters were used to generate Fig. 1, in which the susceptibility to lodging of each barley variety is presented as a function of the average environmental susceptibility to lodging. Predicted values for susceptibility to lodging for each variety under four arbitrarily selected environments are shown in Table 3. Figure 2 presents the model in Eqn (1) plotted against logit axes, showing the individual variety means along the $y$-axis and the environmental means along the $\mathrm{x}$-axis. This results in straight lines analogous to the sensitivity analysis of continuous yield variables (Öfversten et al. 2002). Correlations between the estimated parameters and the varietal properties are shown in Table 4.

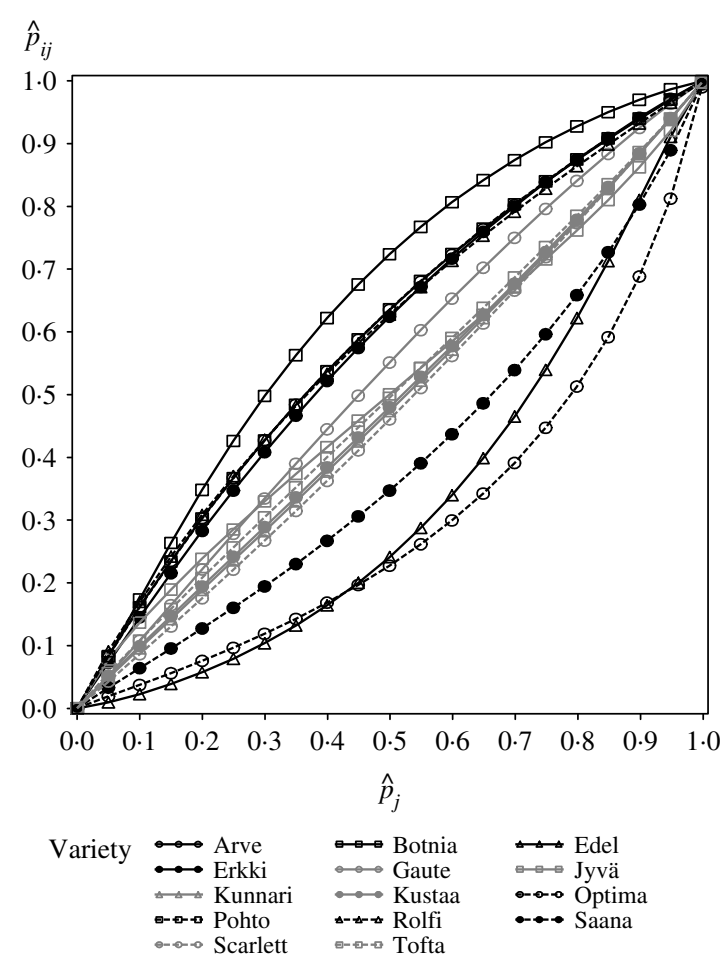

Fig. 1. Susceptibility to lodging of barley varieties as a function of the mean environmental susceptibility to lodging.

It is important to note that the susceptibility to lodging of each barley variety depends on two components: a constant level (measured by $\hat{g}_{i}$ ) reflecting the susceptibility of a variety to lodging in average 
Table 3. Predicted susceptibility to lodging of barley varieties under four arbitrary environments

\begin{tabular}{|c|c|c|c|c|}
\hline \multirow[b]{2}{*}{ Barley variety } & \multicolumn{4}{|c|}{ Environmental susceptibility } \\
\hline & $\hat{p}_{i, 0 \cdot 25}$ & $\hat{p}_{i, 0 \cdot 38}$ & $\hat{p}_{i, 0 \cdot 5}$ & $\hat{p}_{i, \mathbf{0} \cdot 75}$ \\
\hline Arve & $0 \cdot 37$ & $0 \cdot 51$ & $0 \cdot 64$ & $0 \cdot 84$ \\
\hline Botnia & $0 \cdot 43$ & $0 \cdot 60$ & 0.72 & $0 \cdot 90$ \\
\hline Edel & $0 \cdot 08$ & $0 \cdot 15$ & $0 \cdot 24$ & $0 \cdot 54$ \\
\hline Erkki & $0 \cdot 35$ & $0 \cdot 50$ & 0.63 & $0 \cdot 84$ \\
\hline Gaute & $0 \cdot 28$ & $0 \cdot 42$ & 0.55 & $0 \cdot 80$ \\
\hline Jyvä & $0 \cdot 29$ & $0 \cdot 40$ & $0 \cdot 50$ & $0 \cdot 72$ \\
\hline Kunnari & $0 \cdot 24$ & $0 \cdot 36$ & $0 \cdot 47$ & 0.73 \\
\hline Kustaa & $0 \cdot 24$ & $0 \cdot 36$ & $0 \cdot 48$ & 0.73 \\
\hline Optima & $0 \cdot 10$ & $0 \cdot 16$ & $0 \cdot 23$ & $0 \cdot 45$ \\
\hline Pohto & $0 \cdot 37$ & $0 \cdot 51$ & 0.64 & $0 \cdot 84$ \\
\hline Rolfi & $0 \cdot 37$ & $0 \cdot 51$ & 0.63 & $0 \cdot 83$ \\
\hline Saana & $0 \cdot 16$ & $0 \cdot 25$ & $0 \cdot 35$ & $0 \cdot 60$ \\
\hline Scarlett & $0 \cdot 22$ & $0 \cdot 34$ & $0 \cdot 46$ & 0.72 \\
\hline Tofta & $0 \cdot 26$ & $0 \cdot 38$ & $0 \cdot 50$ & $0 \cdot 74$ \\
\hline
\end{tabular}

Environmental susceptibilities to lodging: $0 \cdot 25,0 \cdot 38$ (average environment), 0.5 and $0 \cdot 75$, respectively.

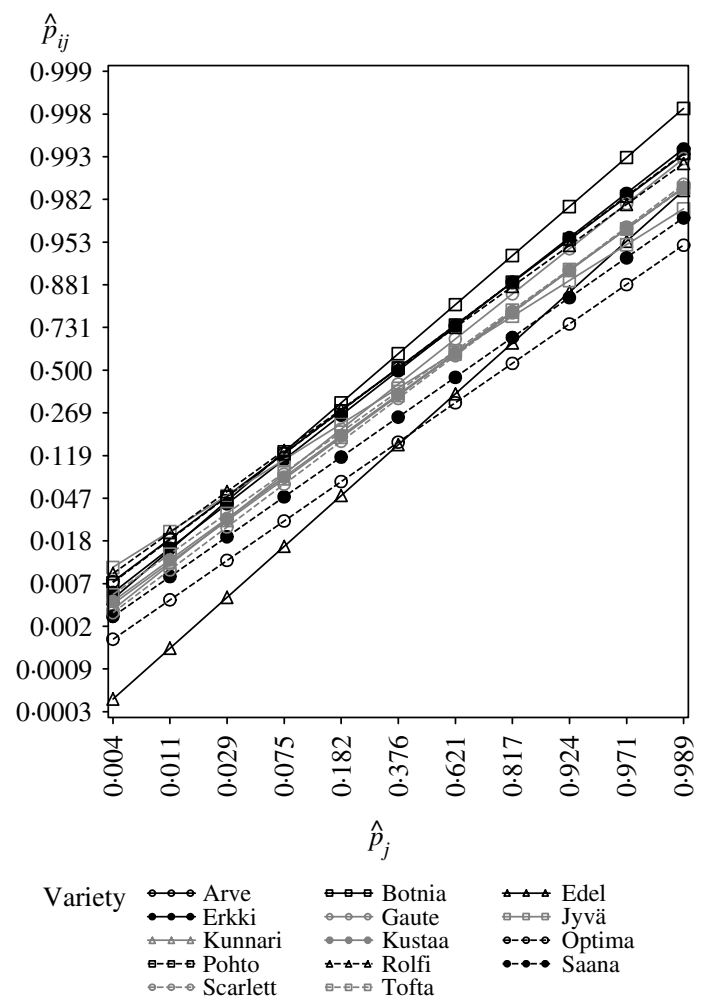

Fig. 2. Linear representation of the susceptibility to lodging of barley varieties as a function of the mean environmental susceptibility to lodging.
Table 4. Correlations between estimated parameters and varietal properties

\begin{tabular}{|c|c|c|c|c|}
\hline & $\hat{g}_{i}$ & $\hat{b_{i}}$ & $\begin{array}{l}\text { Height } \\
(\mathrm{cm})\end{array}$ & $\begin{array}{c}\text { Growing } \\
\text { cycle } \\
\text { (days) }\end{array}$ \\
\hline$\hat{g}_{i}$ & $1 \cdot 000$ & $\begin{array}{l}0 \cdot 036 \\
(P=0 \cdot 90)\end{array}$ & $\begin{array}{l}0.158 \\
(P<0 \cdot 001)\end{array}$ & $\begin{array}{l}-0 \cdot 252 \\
(P<0 \cdot 001)\end{array}$ \\
\hline$\hat{b_{i}}$ & & $1 \cdot 000$ & $\begin{array}{l}0 \cdot 195 \\
(P<0 \cdot 001)\end{array}$ & $\begin{array}{l}-0.049 \\
(P=0 \cdot 17)\end{array}$ \\
\hline Height $(\mathrm{cm})$ & & & $1 \cdot 000$ & $\begin{array}{l}0 \cdot 227 \\
(P<0.001)\end{array}$ \\
\hline $\begin{array}{l}\text { Growing cycle } \\
\text { (days) }\end{array}$ & & & & 1.000 \\
\hline
\end{tabular}

environmental conditions, and a variable component (measured by $\hat{b_{i}}$ ) which reflects the susceptibility of the same variety to lodging in the actual growing conditions. These two parameters represent the linear relationship between the latent environmental variable $w_{j}$ and the logit of the susceptibility to lodging (lodging probability), with $g_{i}$ and $b_{i}$ being the intercept and slope, respectively, for variety $i$. Using the estimated parameters $\hat{b_{i}}$, varieties can be singled out into sensitive and robust varieties. A $\hat{b}_{i}$ value of one represents a variety that has an average sensitivity for environmental variation. A variety associated to a $\hat{b_{i}}<1$ is more robust than average and a variety associated to a $\hat{b_{i}}>1$ is more sensitive than average. Differences in sensitivity between varieties can best be visualized in the linear representation outlined in Fig. 2 as differences between the slopes. The statistical significances of varietal slopes compared to unit slope are presented in Table 2. Values of $\hat{g}_{i}$ represent the average susceptibility to lodging (lodging probability) on a logit scale.

The results can be used to rank barley varieties according to their susceptibility to lodging in a given environment. The optimum choice of variety depends on the environment. For example, Table 3 shows that Edel $\left(\hat{p}_{i, 0 \cdot 25}=0 \cdot 079\right)$ is the most suitable for favourable conditions, whereas Optima $\left(\hat{p}_{i, \mathbf{0} \cdot 75}=0 \cdot 45\right)$ is the best choice under unfavourable conditions. Figures 1 and 2 allow for more comprehensive comparisons. For example, when environmental susceptibility to lodging is lower than $0 \cdot 45$, Edel represents the most suitable variety, but Optima is the best choice elsewhere. Furthermore, among all the varieties Botnia has the highest susceptibility to lodging uniformly in all environments.

Correlations (Table 4) between the estimated parameters $\left(\hat{b}_{i}\right.$ and $\left.\hat{g}_{i}\right)$ and the varietal properties (height and growing cycle) are in accordance with previous reports and indicate that tall varieties tend to be more sensitive to environmental variation with respect to the susceptibility to lodging. On the other 


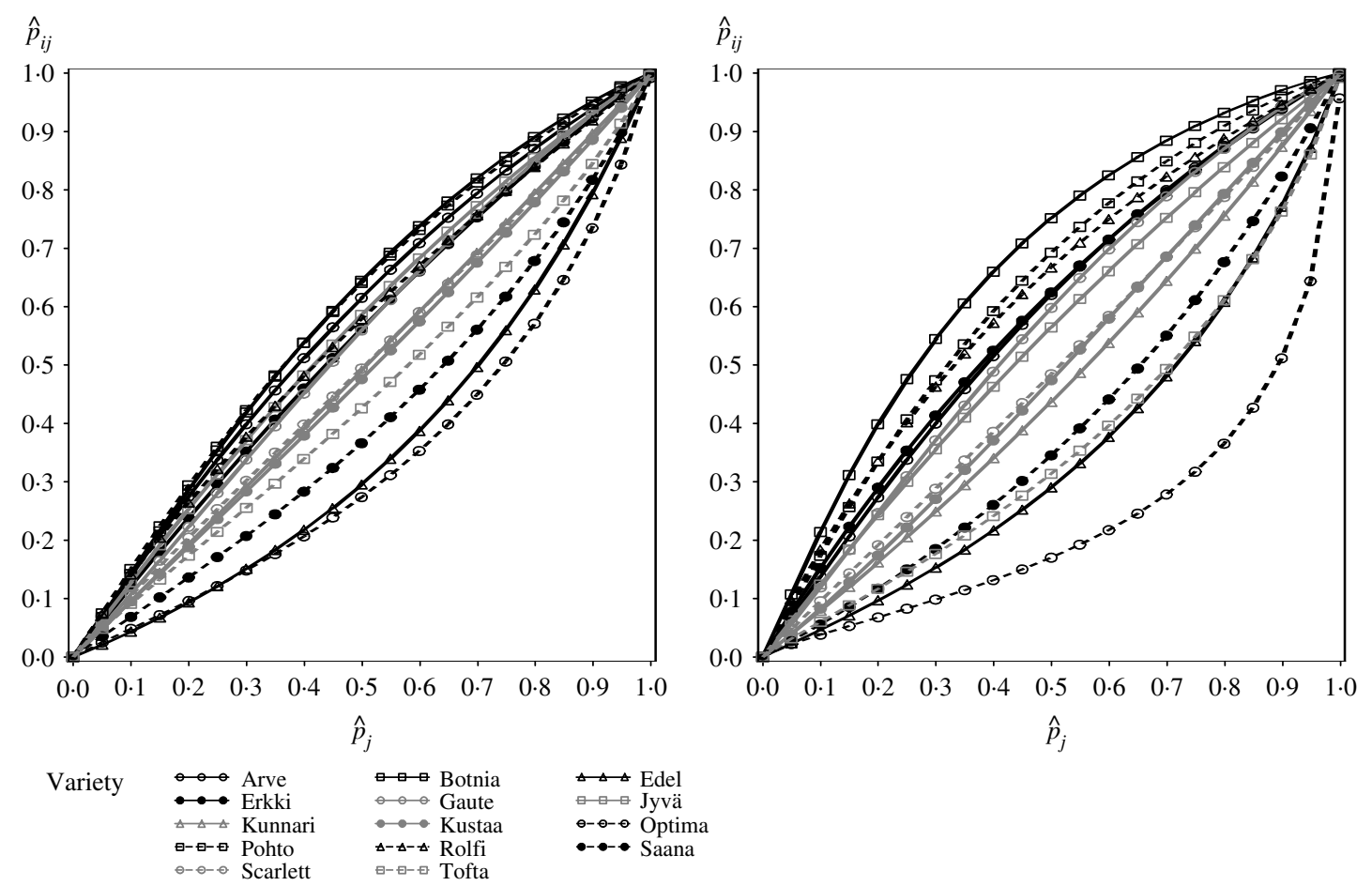

Fig. 3. Susceptibility to lodging of barley varieties as a function of the mean environmental susceptibility to lodging using different lodging thresholds of $0 \cdot 05$ and $0 \cdot 15$ (left and right panel, respectively).

hand, shorter varieties and varieties with an extended growing cycle tend to have a lower overall susceptibility to lodging.

\section{DISCUSSION}

In the current study, the different susceptibilities of barley varieties to lodge under variable environmental conditions was examined. In addition, the sensitivities of barley varieties to environmental variation in terms of susceptibility to lodging were compared. The results obtained allow the prediction of the susceptibility of a particular barley variety to lodging in a given environment. This represents an invaluable tool for identifying barley varieties that are robust to changes in environmental conditions.

As an explanatory variable, the average level of susceptibility to lodging in a given environment was used. This is a latent variable that combines the effects of all external conditions associated to that environment. A similar approach has been used in previous studies (Piepho 1997; Öfversten et al. 2002). The use of the proposed latent variable is justified, because there is no single index available containing sufficient information of the environment as a measurable entity (Eberhart \& Russell 1966). The use of the latent environmental variable approach ensures the usability of the results from the producers' perspective, since they only have to assess the average susceptibility to lodging in their own fields, rather than having to measure a range of related environmental parameters.

For modelling and estimation purposes, analogical results developed to predict the local yielding performance of spring wheat varieties were used (Öfversten et al. 2002). These methods were extended to suit the framework of generalized linear mixed models with logit-link. This permitted the susceptibility to lodging measured on a non-normal binary scale to be evaluated.

The results reflecting the different susceptibilities to lodging of barley varieties are important, but the approach used is equally valuable since it can also be applied for other crops and other traits. Other traits that can be measured on a dichotomous scale include susceptibility to disease or winter damage. Such dichotomous measurements often transform into risk assessment situations. For example, in examining yielding performance, it is often more critical to attain a certain yield threshold, rather than yielding per se (Fox et al. 1997; Piepho \& McCulloch 2004).

An observed proportion of $0 \cdot 10$ of lodging was used as the threshold for significant lodging. Figure 3 presents two graphs similar to that in Fig. 1, derived 
using two different arbitrary thresholds for significant lodging. In the left panel, a limit of 0.05 was applied and in the right panel a value of 0.15 was used. Even though applying different limits changes the scale of the differences between varieties, the ranking of varieties remains essentially the same. The ranking of the varieties is the primary focus, and therefore the choice of the threshold applied is of much less importance.

However, if there is a need to examine differences between varieties in more detail, the limits imposed have to be considered carefully. Some critical thresholds of winter damage, viral infections and foliar diseases of cereals with respect to yield have been defined (Peltonen 1993). A similar approach could be useful when attempting to define more precise criteria for different performance characteristics.

The current research indicates that while differences in sensitivities of susceptibility to lodging can statistically be shown to be only marginally significant more notable differences amongst varieties exist in the overall level of susceptibility to lodging (Table 2). Within the nonlinear framework (logit) used, the clearest inference can therefore be made if both parameters $\left(\hat{b}_{i}\right.$ and $\left.\hat{g}_{i}\right)$ are assessed simultaneously, i.e. the predicted susceptibility to lodging of each variety is assessed in a given environment, which is possible using Figs 1 and 2 and Table 3. That kind of reasoning shows that in many cases, differences between the varieties are practically relevant. In the future, methods for the formal testing of local predictions are required. Öfversten et al. (2002) have evaluated predictions using linear models, but the testing of current predictions is more complicated and requires further work. It may be possible to develop computer simulation or model based testing methods for this purpose.

The authors wish to acknowledge the contribution of the Editor and two referees for their constructive comments and suggestions during the revision and evaluation of this manuscript.

\section{REFERENCES}

Agresti, A. (2002). Categorical Data Analysis, 2nd edition. Hoboken, NJ : John Wiley \& Sons.

Collett, D. (2003). Modelling Binary Data, 2nd edition. Boca Raton, FL: Chapman \& Hall/CRC.

Cox, D. R. \& Snell, E. J. (1989). Analysis of Binary Data, 2nd edition. London: Chapman \& Hall.

Eberhart, S. A. \& Russell, W. A. (1966). Stability parameters for comparing varieties. Crop Science 6, $36-40$.

Finlay, K. W. \& Wilkinson, G. N. (1963). The analysis of adaptation in a plant-breeding programme. Australian Journal of Agricultural Research 14, $742-754$

Fox, P. N., Crossa, J. \& Romagosa, I. (1997). Multienvironment testing and genotype $\times$ environment interaction. In Statistical Methods for Plant Variety Evaluation (Eds R. A. Kempton \& P. N. Fox), pp. 117-138. London: Chapman and Hall.

Gogel, B. J., Cullis, B. R. \& Verbyla, A. P. (1995). REML estimation of multiplicative effects in multi-environment variety trials. Biometrics 51, 744-749.

Kangas, A., Laine, A., Niskanen, M., Salo, Y., Vuorinen, M., Jauhiainen, L. \& NikAnder, H. (2005). Results of official variety trials 1997-2004. Agrifood Research Reports 83, MTT Agrifood Research Finland, Jokioinen, 193 pp.

McCullagh, P. \& Nelder, J. A. (1989). Generalized Linear Models, 2nd edition. London: Chapman \& Hall.
McCulloch, C. E. \& Searle, S. R. (2001). Generalized, Linear, and Mixed Models. New York, NY: John Wiley \& Sons.

Öfversten, J. (1998). Assessing sensitivity of agricultural crop varieties. Journal of Agricultural, Biological, and Environmental Statistics 3, 37-47.

Öfversten, J., Jauhiainen, L., Nikander, H. \& Salo, Y. (2002). Assessing and predicting the local performance of spring wheat varieties. Journal of Agricultural Science, Cambridge 139, 397-404.

Oman, S. D. (1991). Multiplicative effects in mixed model analysis of variance. Biometrika 78, 729-739.

Peltonen, J. (1993). Partitioning of injuries caused by winter, fungal diseases and viral infections in cereals. Agricultural Science in Finland 2, 353-355.

PiePHO, H.-P. (1997). Analyzing genotype-environment data by mixed models with multiplicative terms. Biometrics $\mathbf{5 3}$, 761-766.

PiePhO, H.-P. (1998). Methods for comparing the yield stability of cropping systems - a review. Journal of Agronomy and Crop Science 180, 193-213.

Piepho, H.-P. (1999). Stability analysis using the SAS system. Agronomy Journal 91, 154-160.

Piepho, H.-P. \& McCulloch, C. E. (2004). Transformations in mixed models: application to risk analysis for multienvironment trial. Journal of Agricultural, Biological, and Environmental Statistics 9, 123-137.

YAtes, F. \& Cochran, W. G. (1938). The analysis of groups of experiments. Journal of Agricultural Science, Cambridge 28, 556-580. 